\title{
Antioxidant Enzymes and Photosynthetic Responses to Drought Stress of Three Canna edulis Cultivars
}

\author{
Wen-E Zhang ${ }^{1,2}$, Fei Wang ${ }^{1 *}$, Xue-jun Pan ${ }^{2}$, Zhi-guo Tian ${ }^{1}$, and Xiu-ming Zhao ${ }^{1}$ \\ ${ }^{1}$ College of Horticulture, Northwest A\&F University, Yangling, 712100, Shaanxi, People's Republic of China \\ ${ }^{2}$ College of Agriculture, Guizhou University, Guiyang, 550025, Guizhou, People's Republic of China
}

\begin{abstract}
Edible canna is a productive starch source in some tropical and semitropical regions. In these regions, water deficit stress is one of factors that limit the crop yield. In the present study, we investigated seven physiological indexes and photosynthetic responses of three edible canna (Canna edulis Ker.) cultivars ('PLRF', 'Xingyu-1', and 'Xingyu-2') under 35 days drought stress. Our results indicated that drought treatment caused visible wilting symptoms in all cultivars, especially in 'Xingyu-1'. Coupled with the increase of wilting symptoms, relative water content (RWC) and chlorophyll content decreased progressively, malondialdehyde (MDA) content gradually increased, and key antioxidant enzymes such as superoxide dismutase (SOD), peroxidase (POD), catalase (CAT) activities increased first and then decreased in all three cultivars. The effect of water stress was more pronounced in 'Xingyu-1' than in 'PLRF' and 'Xingyu-2', and in lower leaves than in upper leaves. In addition, 35 days drought stress also significantly reduced the photosynthetic capacity. Consistent with antioxidant parameters, photosynthetic changes of 'Xingyu-2' were less than those of the other cultivars under water deficit stress. Drought stress caused a significant increase of water use efficiency (WUE) in 'Xingyu-2', but little in 'PLRF', and obvious decrease in 'Xingyu-1'. These results indicated that 'Xingyu-2' was more tolerant to drought stress than 'PLRF' and 'Xingyu-1' by maintaining lower lipid peroxidation and higher antioxidant enzyme activities.
\end{abstract}

Additional key words: catalase, edible canna, malondialdehyde, peroxidase, superoxide dismutase, water deficit

\section{Introduction}

Eible canna (Canna edulis Ker.) is one of tuber crop food sources, grown in some tropical and semitropical regions where cereal crops are not suitable for growing due to climatic and agronomic reasons. It has been an useful starch source not only for food but also for industry materials (Pérez et al., 1997). In recent years, edible canna is also used as an important source of bio-fuel, since it has high starch content in the rhizome of about 60-70 percent of its dry weight and a high yield of more than 60-75 tons per hectare by fresh weights (Sriroth et al., 2001; Wu et al., 2005). In production, water is essential for plant growth and metabolism (Boyer, 1982; Jones and Corlett, 1992). In tropical and semitropical regions, plants are often exposed to water deficit stress, also known as drought stress, which greatly limits the crop yield by decreasing photosynthesis (Gobin, 2012; Mafakheri et al., 2010). Drought stress may lead to production of excessive photon energy in chloroplasts which damages photosynthetic apparatuses, and this in turn causes a decrease in the photochemical efficiency. Photosynthesis inhibition is one of the primary physiological consequences of drought stress (Chaves, 1991; Cornic, 1994; Lawlor, 1995). The reduction of intercellular $\mathrm{CO}_{2}$ concentration, as a results of stomatal closure, is one of the main causes of photosynthesis decrease under water stress conditions (Chaves and Oliveira, 2004; Ennahli and Earl, 2005; Grassi and Magnani, 2005). On the other hand, under drought stress circumstance, the decrease of chlorophyll content also leads to reduction of the photosynthesis efficiency (Loggini et al., 1999).

In addition to that, drought stress can cause the formation of reactive oxygen species (ROS) as a result of water potential

\footnotetext{
*Comesponding author: xnwangfei521@126.com

※ Received 18 March 2013; Revised 1 July 2013; Accepted 3 July 2013. This work was supported by the Social Welfare Program of Forestry Administration in China (200704009), Scientific and technological project in Shaanxi Province (2009K01-11) and the Natural Science Foundation of Guizhou Province ([2007]2055). The authors thank Technical Bureau of Xingyi, Guizhou Province for assistance to obtain the edible canna bulbs and Prof. Feng Chen (Department of Plant Sciences, University of Tennessee) for proofreading the manuscript. 
alteration in the plant cells (Sanchez-Rodrigues et al., 2010; Türkan et al., 2005). The ROS is potentially harmful to cell membranes, resulting in oxidative degradation of membrane lipids called lipid peroxidation (Foyer et al., 1994; Zhang et al., 2011). Malondialdehyde (MDA) is one of the breakdown products of lipid peroxidation, directly reflect the degree of plant cell damage, and has been frequently used as an indicator of lipid peroxidation in vivo ( $\mathrm{Li}$ et al., 2013).

Plants have developed different scavenging mechanisms to protect against oxidative stress by controlling the level of ROS by interacting network of antioxidant systems, including nonenzymatic antioxidants and antioxidative enzymes, such as ascorbic acid (AsA), glutathione (GSH), catalase (CAT), superoxide dismutase (SOD), and peroxidase (POD) (Habibi, 2012; Ozkur et al., 2009; Xue and Liu, 2008). The SOD can convert superoxide anion to $\mathrm{O}_{2}$ and hydrogen peroxide $\left(\mathrm{H}_{2} \mathrm{O}_{2}\right)$, which can be scavenged by CAT and a variety of peroxidases through the oxidation of co-substrates such as AsA or other antioxidants (Basu et al., 2010). Detailed information on drought-tolerance patterns is important not only for successful cultivation, but also for understanding the antioxidant defense mechanism of tolerant cultivars to abiotic factors and their dynamics in dry lands.

A great deal of work on edible canna has been done in the field of underlying photosynthetic capacity (Kato and Imai, 1996), starch characterization (Lii and Chang, 1991; Moorthy et al., 2002; Pérez et al., 1997, 1998; Thitipraphunkul et al., 2003a, 2003b), assessment of gelatinization parameters (Lares and Pérez, 2006; Wootton and Bamunuarachchi, 1978) and starch nutrition (Pérez and Lares, 2005; Yun et al., 2004). Nevertheless, only little is known about drought tolerance of the edible canna during starch accumulation stage, especially the antioxidantive enzymes responsible for drought stress. This study was conducted to (i) investigate antioxidant enzymes defense mechanism of three edible canna cultivars, Canna edulis Ker. 'PLRF', 'Xingyu-1', and 'Xingyu-2' under drought conditions, (ii) characterize the difference in response to drought stress of three edible canna cultivars at a starch accumulation stage, and (iii) understand the relationship between antioxidant defense mechanism and drought stress. It will provide valuable information for cultivation, breeding for drought-tolerant plants, and deciphering its molecular mechanisms of drought tolerance in arid and semi-arid areas.

\section{Materials and Methods}

\section{Study Site and Plant Materials}

The study was conducted at the Northwest A \& F University $\left(34^{\circ} 283^{\prime} \mathrm{N}, 108^{\circ} 067^{\prime} \mathrm{E}, 560 \mathrm{~m}\right.$ altitude), which is situated in the center part of Kuan-chung Plain in Shaanxi Province,
China. Belonging to an arid and semi-arid region, the mean annual precipitation in this area is about 500 to $800 \mathrm{~mm}$, $60 \%$ of which falls from June to September, with a marked dry season from October to May. In March 2010, bulbs of three edible canna (Canna edulis Ker.) 'PLRF', 'Xingyu-1', and 'Xingyu-2' were obtained from Technical Bureau of Xingyi, Guizhou Province in China. The bulbs were planted in the university nursery. When five euphylla were fully expanded at the latter part of May, the young plants were transplanted into plastic pots $(45 \mathrm{~cm}$ diameter $\times 30 \mathrm{~cm}$ height $)$ that contained $20 \mathrm{~kg}$ soil (yellow loam), screened by $1 \mathrm{~cm}$ sieve. A young plant was transplanted per pot. The transplanted plants were regularly weeded and irrigated about 2-3 times per week until September in a greenhouse, where the mean temperature was $23-30 / 16-19^{\circ} \mathrm{C}$ (day/night), and relative humility (RH) was between 50 and $70 \%$ (from morning to late afternoon) during the experiment. On September 6 (100 days after transplanting), 36 pots with similar plant size were selected and irrigated to the field capacity. From September 6 to October 11, the plant materials were treated under two irrigation regimes: the regular irrigation (control) and 35 days of continuous drought treatment. The soil water potential was monitored using a tensiometer (Tension Meter System, Soilspec, Australia). The soil water potential of the control was continuously monitored to maintain the range from 10 to $30 \mathrm{Kpa}$, which was suitable for the growth of most crops (Adhanom et al., 2012). The soil water potential under drought stress was continuously controlled to maintain above $50 \mathrm{Kpa}$, which is the range, known as drought to most crops (Adhanom et al., 2012). Six potted plants under each irrigation regime per cultivar were used for analysis of physiological measurements. The physiological measurements were taken weekly (7, 14, 21,28 , and 35 days). Samples of every measurement time point were harvested from the same ten leaves in upper and lower positions and pooled for determination of parameters. The plant was divided into two parts (upper and lower part) from the intermediate portion. Each treatment had three replicates and each replicate included 2 pots with one plant in a pot.

\section{Determination of Physiological Parameters}

Relative water content: Leaf water status was estimated in the fully developed leaves by measuring the relative water content (RWC). Ten discs with the diameter about $1 \mathrm{~cm}$ were cut from ten leaves using a hole puncher (3 replicates), and the fresh weight (FW) was immediately taken after harvest. Then the leaf discs were placed in a petri dish $(90$ $\mathrm{mm}$ in diameter) filled with MilliQ distilled water overnight at room temperature and the turgid fresh weight (TW) for each replicate was measured. The samples were dried in an 
oven at $80^{\circ} \mathrm{C}$ for $48 \mathrm{~h}$ to measure their dry weights (DW). The relative water content was calculated as Weatherley's meathod (Weatherley, 1950): RWC $(\%)=(F W-D W) /(T W$ - DW) $\times 100$.

\section{Determination of photosynthetic gas exchange parameters:}

Before harvest, the net photosynthetic rate (Pn), stomatal conductance (Cond), intercellular carbon dioxide concentration (Ci), and transpiration rate $(\mathrm{Tr})$ were measured on October 11 using a photosynthetic system (Li-6400, Li-Cor, Inc., Nebraska, USA). The measurements were taken from three most recently matured leaves in each replicate plant during the period from 9:00 to 11:00 in the morning, under photosynthetic flux density (PPFD) of $1,000 \mu \mathrm{mol} \cdot \mathrm{m}^{-2} \cdot \mathrm{s}^{-1}$ provided by a LED light source. The air humidity in the leaf chamber was about $50 \%$, with $\mathrm{CO}_{2}$ concentration of $420-450 \mu \mathrm{mol} \cdot \mathrm{mol}^{-1}$, ambient air temperature of $24 \pm 2{ }^{\circ} \mathrm{C}$, and flow rate of $500 \mu \mathrm{mol} \cdot \mathrm{s}^{-1}$. Water use efficiency (WUE) was calculated as previously described by Pan et al. (2011): WUE $=\mathrm{Pn} / \mathrm{Tr}$.

Pigments analysis: The $0.02 \mathrm{~g}(\mathrm{~d}=0.0001)$ of shredded leaves were randomly taken from ten upper or lower leaves of plants (3 replicates). Chlorophyll a (Chl.a) and chlorophyll b (Chl.b) of the leaves were extracted in $5 \mathrm{~mL} 80 \%(\mathrm{v} / \mathrm{v})$ acetone, and the contents of pigment were determined by measuring absorbance at $663 \mathrm{~nm}$ and $645 \mathrm{~nm}$, using a UV-Vis spectro-photometer (UV-1700, Shimadzu, Kyoto, Japan). The Chl.a and Chl.b contents were calculated according to Lichtenthaler and Wellburn (1983).

\section{Assays of SOD, CAT, POD activities and MDA contents:}

For extraction of enzymes, leaf samples (0.5 g) were homogenized over ice in $8 \mathrm{~mL}$ ice-cold sodium phosphate buffer (PBS, $50 \mathrm{mM}, \mathrm{pH}$ 7.8) containing 1\% (w/v) polyvinyl polypyrolidone (PVP). The homogenate was centrifuged at $15,000 \mathrm{~g}$ for $20 \mathrm{~min}$ at $4^{\circ} \mathrm{C}$, and the supernatant was divided into $500 \mu \mathrm{L}$ aliquots and stored at $-70^{\circ} \mathrm{C}$ as crude extract for enzyme activity and MDA content assay.

Activities of superoxide dismutase (SOD, EC 1.15.1.1) were determined by measuring its ability to inhibit the photo-reduction of nitroblue tetrazolium (NBT) (Giannopolities and Ries, 1977) based on the absorbance at $560 \mathrm{~nm}$. Activities of catalase (CAT, EC 1.11.1.6) were assayed by measuring the decrease in absorption at $240 \mathrm{~nm}$ in $3 \mathrm{~mL}$ reaction medium containing $2.85 \mathrm{~mL}$ PBS (50 mM, pH 7.0), $50 \mu \mathrm{L}$ $\mathrm{H}_{2} \mathrm{O}_{2}(10 \mathrm{mM})$ and $100 \mu \mathrm{L}$ enzyme extract as described by Chance and Maehly (1955). For measurement peroxidase (POD, EC 1.11.1.7) activity, $50 \mu \mathrm{L}$ of the supernatant was mixed with $2.95 \mathrm{~mL}$ PBS (100 mM, pH 6.8) containing $1.65 \mu \mathrm{L}$ guaiacol and $0.56 \mu \mathrm{L} 30 \% \mathrm{H}_{2} \mathrm{O}_{2}$ according to $\mathrm{Li}$ et al. (2009). Reaction started by addition of enzyme extract in phosphate buffer. The increase of the solution absorbance was recorded at $470 \mathrm{~nm}$ within $6 \mathrm{~min}$ by an UV-Vis spectrophotometer (UV-1700, Shimadzu, Kyoto, Japan). One unit (U) of SOD activity was defined as the amount of enzyme required to cause $50 \%$ inhibition of the NBT photo-reduction rate per hour. One CAT activity unit (U) was defined as absorbance at $240 \mathrm{~nm}$ decreasing 0.01 per minute and one POD unit activity was defined as absorbance at $470 \mathrm{~nm}$ increasing 0.1 per minute. The activities of SOD, CAT, and POD were expressed as units per gram of fresh weight.

The MDA contents were analyzed according to Heath and Packer (1968) with slight modification, for taking into account the possible influence of interfering compounds in the assay for thiobarbituric acid (TBA) reactive substances. $1 \mathrm{~mL}$ of supernatant and $2 \mathrm{~mL}$ of $10 \%(\mathrm{w} / \mathrm{v})$ trichloroacetic acid (TCA) containing $0.6 \%(\mathrm{w} / \mathrm{v})$ TBA were added. After heating at $100^{\circ} \mathrm{C}$ for $15 \mathrm{~min}$, the mixture was quickly cooled in ice-water mixture and centrifuged at 4,000 $\mathrm{g}$ for $10 \mathrm{~min}$. The absorbance of the supernatant was measured at $532 \mathrm{~nm}$ and the value for non specific absorption at $600 \mathrm{~nm}$ and $450 \mathrm{~nm}$ was subtracted. The contents of MDA were expressed as nmol MDA per gram fresh weight with an extinction coefficient of $155 \mathrm{mM} \cdot \mathrm{cm}^{-1}$ and the coefficient at $450 \mathrm{~nm}$ according to $\mathrm{Li}$ et al. (2009) was 0.56: MDA concentration $\left(\mathrm{nmol} \cdot \mathrm{mL}^{-1}\right)=6.45\left(\mathrm{~A}_{532}-\mathrm{A}_{600}\right)-0.56 \mathrm{~A}_{450}$.

\section{Statistical Analysis}

The treatment means were compared according to Duncan's multiple range tests at 0.05 level using SAS 8.1 statistical software.

\section{Results}

\section{Plant Growth}

As shown in Figs. 1A to 1R, significant drought symptoms could be observed in all cultivars as yellowing, brown spot, and necrosis under drought stress. But the wilting symptoms were more severe and earlier in 'PLRF' (Figs. 1A to 1F) and 'Xingyu-1' (Figs. 1G to $1 \mathrm{~L}$ ) than in 'Xingyu-2' (Figs. $1 \mathrm{M}$ to $1 \mathrm{R}$ ), and in lower leaves than in upper leaves. Wilting symptoms were not obvious in the upper leaves of three cultivars after 7 days of drought stress (Figs. 1A to $1 \mathrm{~B}, 1 \mathrm{G}$ to $1 \mathrm{H}$, and $1 \mathrm{M}$ to $1 \mathrm{~N}$ ), but a sharp increase of wilting symptom was observed in lower leaves of 'PLRF' and 'Xingyu-1' under 7 days drought stress (Figs. $1 \mathrm{~B}$ and $1 \mathrm{H}$ ). Since then, the wilting index continued to increase. 'Xingyu-1' (Figs. 1G to $1 \mathrm{~L}$ ) was more serious than 'PLRF' (Figs. 1A to $1 \mathrm{~F}$ ), when the drought stress continued to 35 days, the lower leaves of 'Xingyu-1' wilted seriously and was even unsuitable to 


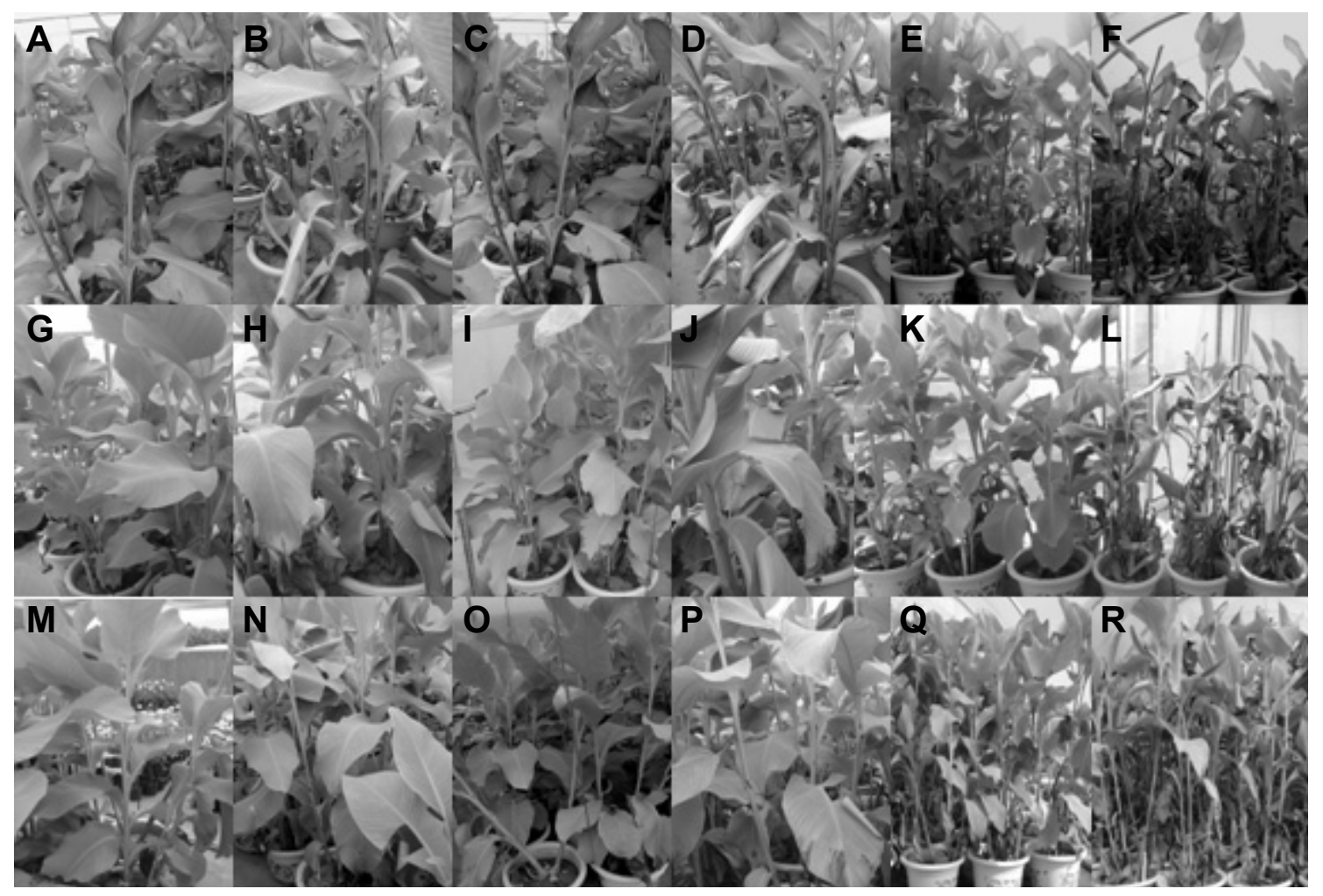

Fig. 1. Growth of edible canna 'PLRF' (A-F), 'Xingyu-1' (G-L), and 'Xingyu-2' (M-R) under drought stress for 14 (B, H, and $N$ ), $21(D, J$, and $P)$, or $35(F, L$, and $R)$ days or the control $(A, C, E, G, I, K, M, O$, and $Q)$.

take samples for assay (Fig. 1F). For 'Xingyu-2' (Fig. 1M to $1 \mathrm{R})$, wilting index changed little in upper leaves from 7 days to 28 days drought stress, but it changed obviously from 28 days to 35 days. This trend was more obvious in the lower leaves. As experiment time was longer and this time was a transitional season from summer to autumn, environment temperature and light condition changed largely, leaves of the control were also yellowing, in particular in the lower leaves from 4 Oct to 11 Oct (Figs. 1A, 1C, 1E, $1 \mathrm{G}, 1 \mathrm{I}, 1 \mathrm{~K}, 1 \mathrm{M}, 1 \mathrm{O}$, and $1 \mathrm{Q})$.

\section{Relative Water Content}

In our study, relative water content (RWC) of three canna cultivars progressively decreased under drought stress compared to control (Figs. 2A to 2F). The decrease of RWC was more serious in the lower leaves than in the upper leaves. Among three cultivars, the strongest and earliest decline in RWC was found in 'Xingyu-1' (Figs. 2B and 2E), followed by 'PLRF' (Figs. 2A and 2D), the changes of 'Xingyu-2' under drought stress was the least (Figs. $2 \mathrm{C}$ and $2 \mathrm{~F}$ ). Compared with the control, the RWC decreased significantly in the upper leaves after 28 days, 35 days and 35 days of drought stress in 'Xingyu-1' (Fig. 2B), 'PLRF' (Fig. 2A) and 'Xingyu-2' (Fig. 2C), while that of the lower leaves decreased significantly on 21 days in 'Xingyu-1' (Fig. 2E) and 'PLRF' (Fig. 2D),

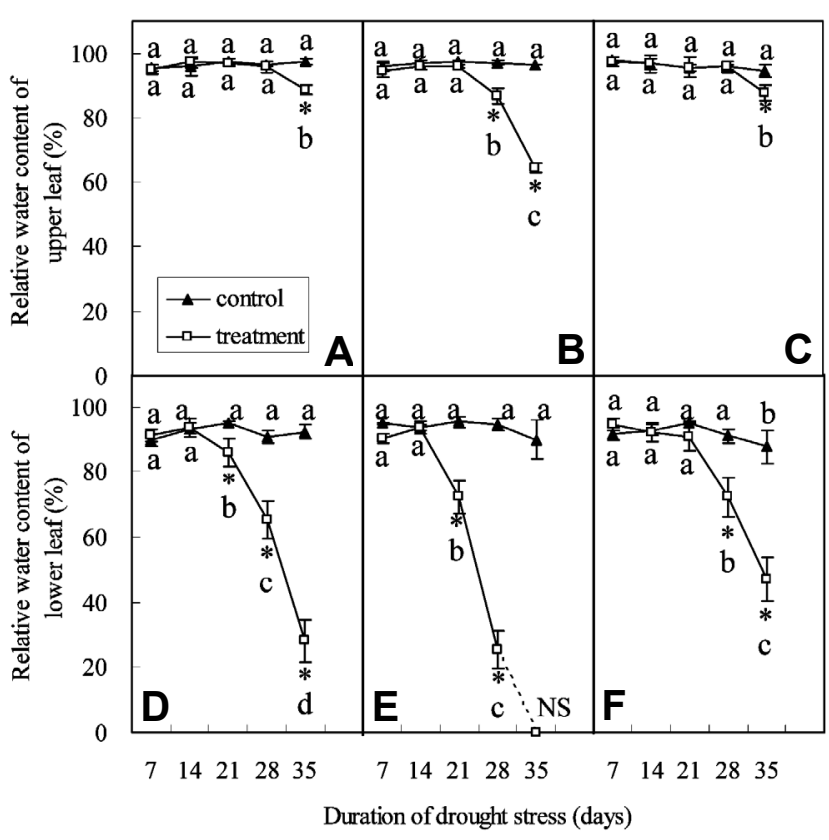

Fig. 2. Effects of drought stress on relative water content in upper leaf $(A-C)$ and lower leaf (D-F) of edible canna 'PLRF' $(A D)$, 'Xingyu-1' (BE), and 'Xingyu-2' (CF). Different letters on top of error bars represent significant differences among every measurement time point according to Duncan's multiple range tests and asterisks represent significant difference as compared to the control at $p<0.05(n=3)$. The NS represents unable to take samples at the time point. Vertical error bars represent SD. 
and 28 days in 'Xingyu-2' (Fig. 2F), respectively. The lower leaves of 'Xingyu-1' wilted seriously on 28 days of drought stress, the RWC decreased to $25.36 \%$ at this point time, and the lower leave of this cultivar completely shriveled on 35 days of drought stress, and was unsuitable to take samples (Fig. 1E).

\section{Photosynthetic Parameters}

As a consequence of decrease of RWC under water stress, a sharp decline in the rate of net photosynthesis (Pn), the value of stomatal conductance (Cond), intercellular carbon dioxide concentration $(\mathrm{Ci})$, and transpiration rate $(\mathrm{Tr})$ was observed in three cultivars, in addition to $\mathrm{Ci}$ of 'Xingyu-1' (Table 1). The decrease of Pn, Cond, $\mathrm{Ci}$ and $\mathrm{Tr}$ in 'PLRF' was stronger than in 'Xingyu-1' and 'Xingyu-2' after 35 days of continuous drought stress. Compared to the control, the water use efficiency (WUE) decreased slightly in 'PLRF', decreased obviously in 'Xingyu-1', while increased significantly in 'Xingyu-2' under 35 days drought stress (Table 1).

\section{Photosynthetic Pigments Content}

In upper leaves, compared with the control, chlorophyll a (Chl.a) contents decreased significantly in 'PLRF' and 'Xingyu-1' after 35 days of drought stress (Figs. 3A and 3B), while Chl.a content of 'Xingyu-2' significantly increased at 7 days, 21 days and 28 days, and there were no significant difference at 14 days and 35 days between the control and drought stress (Fig. 3C). In lower leaves, compared with the control, drought stress obviously declined Chl.a content of 'PLRF' and 'Xingyu-1' from 7 days to 35 days, except

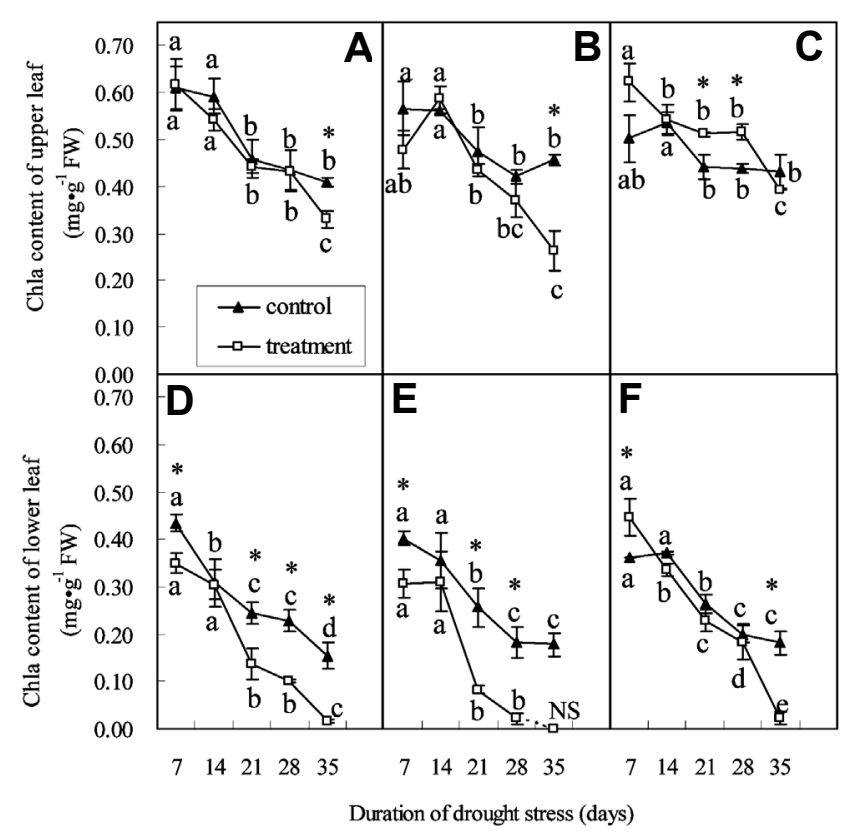

Fig. 3. Effects of drought stress on Chl.a content in upper leaf $(A-C)$ and lower leaf (D-F) of edible canna 'PLRF' (AD), 'Xingyu-1' (BE), and 'Xingyu-2' (CF).

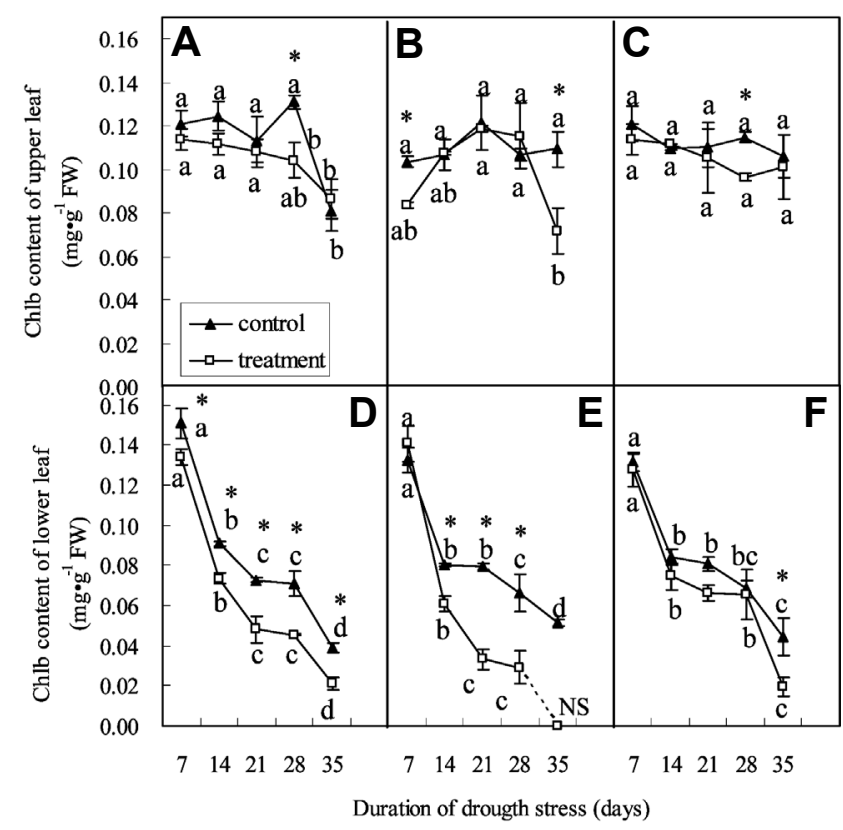

Fig. 4. Effects of drought stress on Chl.b content in upper leaf (A-C) and lower leaf (D-F) of edible canna 'PLRF' (AD), 'Xingyu-1' (BE), and 'Xingyu-2' (CF).

Table 1. Net photosynthetic rate $(\mathrm{Pn})$, stomatal conductance (Cond), intercellular $\mathrm{CO}_{2}$ concentration (Ci), transpiration rate (Tr), and water use efficiency (WUE) in edible canna 'PLRF', 'Xingyu-1', and 'Xingyu-2' under 35 days of drought stress.

\begin{tabular}{lccccccc}
\hline \hline Cultivar & Treatment & $\begin{array}{c}\mathrm{Pn} \\
\left(\mu \mathrm{mol} \mathrm{CO} \cdot \mathrm{m}^{-2} \cdot \mathrm{s}^{-1}\right)\end{array}$ & $\begin{array}{c}\mathrm{Cond} \\
\left(\mathrm{mol} \mathrm{HO}_{2} \cdot \mathrm{m}^{-2} \cdot \mathrm{s}^{-1}\right)\end{array}$ & $\begin{array}{c}\mathrm{Ci} \\
\left(\mu \mathrm{mol} \mathrm{CO}_{2} \cdot \mathrm{mol}^{-1}\right)\end{array}$ & $\begin{array}{c}\mathrm{Tr} \\
\left(\mathrm{mmol} \mathrm{H}_{2} \mathrm{O} \cdot \mathrm{m}^{-2} \cdot \mathrm{s}^{-1}\right)\end{array}$ & $\begin{array}{c}\mathrm{WUE} \\
\left(\mu \mathrm{mol} \mathrm{CO}_{2} \cdot \mathrm{mmol}^{-1} \mathrm{H}_{2} \mathrm{O}\right)\end{array}$ \\
\hline PLRF & Control & $9.54 \pm 2.02 \mathrm{a}^{\mathrm{z}}$ & $0.099 \pm 0.005 \mathrm{a}$ & $217.19 \pm 9.98 \mathrm{a}$ & $2.107 \pm 0.443 \mathrm{a}$ & $4.53 \pm 0.006 \mathrm{a}$ \\
& Drought & $0.74 \pm 0.24 \mathrm{~b}$ & $0.007 \pm 0.002 \mathrm{~b}$ & $157.13 \pm 10.95 \mathrm{~b}$ & $0.203 \pm 0.059 \mathrm{~b}$ & $3.96 \pm 0.356 \mathrm{a}$ \\
\multirow{2}{*}{ Xingyu-1 } & Control & $8.89 \pm 0.75 \mathrm{a}$ & $0.118 \pm 0.004 \mathrm{a}$ & $292.64 \pm 6.95 \mathrm{a}$ & $1.823 \pm 0.1597 \mathrm{a}$ & $4.82 \pm 0.409 \mathrm{a}$ \\
& Drought & $1.54 \pm 0.41 \mathrm{~b}$ & $0.019 \pm 0.007 \mathrm{~b}$ & $274.04 \pm 14.00 \mathrm{a}$ & $0.463 \pm 0.06 \mathrm{~b}$ & $3.45 \pm 0.417 \mathrm{~b}$ \\
\multirow{2}{*}{ Xingyu-2 } & Control & $11.96 \pm 2.04 \mathrm{a}$ & $0.145 \pm 0.020 \mathrm{a}$ & $243.77 \pm 16.45 \mathrm{a}$ & $2.933 \pm 0.198 \mathrm{a}$ & $4.06 \pm 0.489 \mathrm{~b}$ \\
& Drought & $2.35 \pm 0.03 \mathrm{~b}$ & $0.011 \pm 0.002 \mathrm{~b}$ & $51.99 \pm 10.00 \mathrm{~b}$ & $0.247 \pm 0.035 \mathrm{~b}$ & $9.90 \pm 0.357 \mathrm{a}$ \\
\hline
\end{tabular}

${ }^{z}$ Data are mean of three replicates \pm SD. Different letters indicate statistical differences between drought treatment and the control in the same cultivar at $p<0.05(\mathrm{n}=3)$ according to Duncan's multiple range tests. 
14 days (Figs. 3D and 3E). But Chl.a contents of 'Xingyu-2' increased obviously at 7 days of drought stress, and there was no significant difference between the control and drought stress from 14 days to 28 days, the Chl.a content decreased obviously only after 35 days of drought stress (Fig. 3F).

In upper leaves, no significant difference of chlorophyll b (Chl.b) contents was detected in 'PLRF' and 'Xingyu-2' between the control and drought stress from 7 days to 35 days, except for 28 days (Figs. 4A and 4C), but drought stress obviously decreased Chl.b contents of 'Xingyu-1' at 7 days and 35 days (Figs. 4B). In lower leaves, the Chl.b contents of 'PLRF' and 'Xingyu-1' remarkably declined under drought stress from 7 days to 35 days when compared with the control, except 'Xingyu-1' at 7 days (Figs. 4D and 4E). However, Chl.b contents of 'Xingyu-2' in lower leaves sharply reduced by $55.36 \%$ only at 35 days of drought stress (Fig. 4F).

\section{Malondialdehyde Content}

For three cultivars, malondialdehyde (MDA) contents were progressively increased during experiment period in the test leaves (Figs. 5A and 5F). In the same cultivar, increase of MDA contents was stronger in the lower leaves than in the upper leaves. Compared with the control, continuous drought stress could considerably aggravate the membrane lipids peroxidation, the MDA contents of all cultivars in lower leaves increased significantly from 28 days to 35 days. The maximum increase rate of MDA contents was observed in the 'Xingyu-1', followed by 'PLRF', and then 'Xingyu-2'.

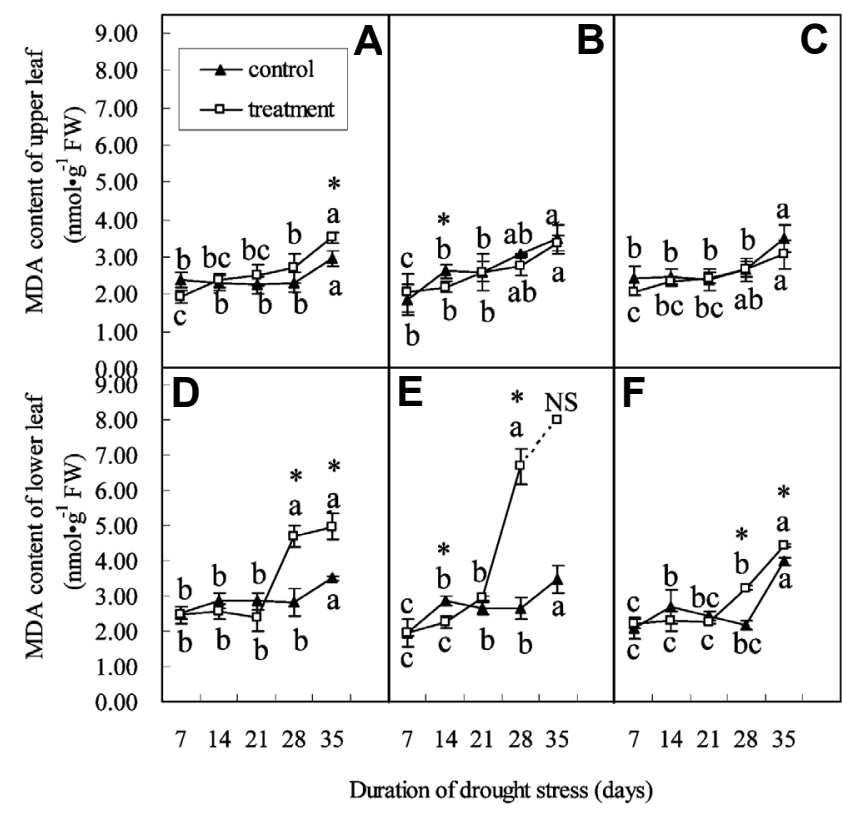

Fig. 5. Effects of drought stress on MDA content in upper leaf (A-C) and lower leaf (D-F) of edible canna 'PLRF' (AD), 'Xingyu-1' (BE), and 'Xingyu-2' (CF).

\section{Antioxidant Enzyme Activities}

Being exposed to drought stress, the antioxidant enzymes in three edible canna cultivars showed different responses (Figs. 6A to 6F). During whole experiment period, the activity of superoxide dismutase (SOD) increased progressively first and then changed little or decreased slightly in upper leaves of three cultivars (Figs. 6A to 6C). In upper leaves, the SOD activities of 'PLRF' under both treatments and 'Xingyu-2' in the control were peaked at 21 days, but these of 'Xingyu-1' under both treatments and 'Xingyu-2' under drought stress reached peak at 28 days (Figs. 6A to $6 \mathrm{C}$ ). There was significant difference of SOD activity in lower leaves of three cultivars between the control and drought stress (Figs. 6D to 6F). Compared with the control, drought stress significantly depressed SOD activity of 'PLRF' from 7 days to 35 days, but these of 'Xingyu-1' and 'Xingyu-2' were no significant difference between the control and drought stress.

In upper leaves of 'PLRF' and 'Xingyu-2', catalase (CAT) activity showed little difference between both treatments from 7 days to 35 days, in addition to that of 'Xingyu-2' under 35 days of drought stress was obviously lower than that of the control (Figs. 7A and 7C), but drought stress significantly reduced CAT activity of 'Xingyu-1' in upper leaves from 7 days to 35 days compared with the control (Fig. 7B). In the lower leaves, in contrasted with the control, drought stress obviously enhanced CAT activity in all cultivars at 7 days (Figs. 7D to 7F). Since then, CAT activity of 'PLRF' declined sharply first and then kept stable (Fig. 7D), that of 'Xingyu-1'

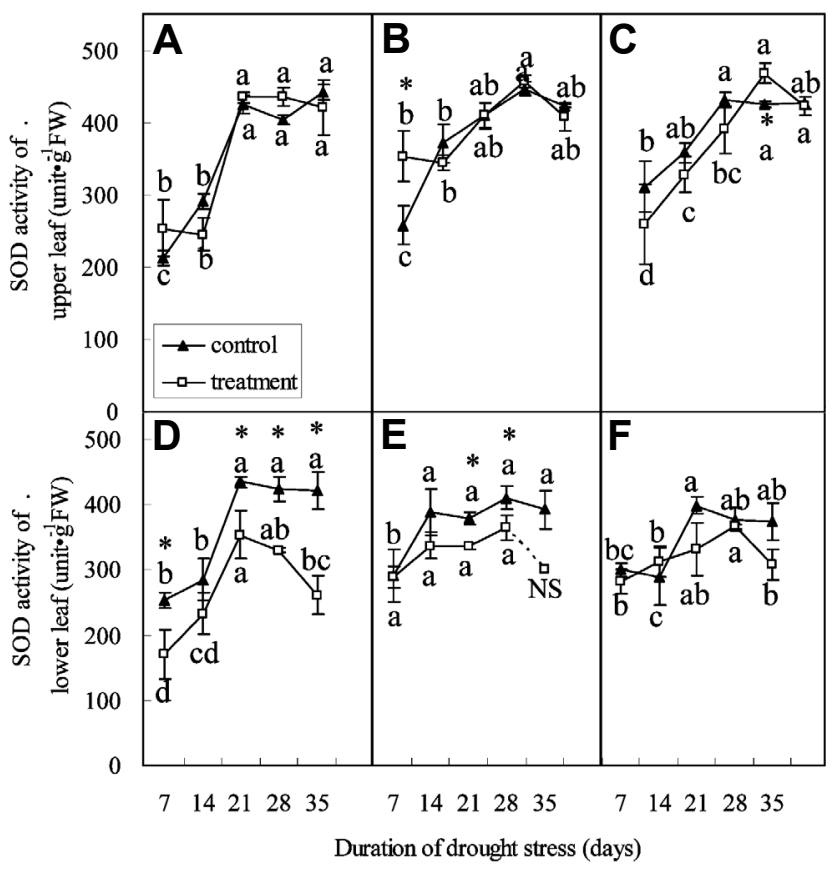

Fig. 6. Effects of drought stress on SOD activity in upper leaf $(A-C)$ and lower leaf (D-F) of edible canna 'PLRF' (AD), 'Xingyu-1' (BE), and 'Xingyu-2' (CF). 
progressively dropped (Fig. 7E), while that of 'Xingyu-2' kept stable first and then declined sharply (Fig. 7F). The CAT activities under drought stress were significantly lower than those of the control from 14 days to 35 days in 'PLRF', from 21 days to 35 days in 'Xingyu-1', and from 29 days to 35 days in 'Xingyu-2', respectively (Figs. 7D to 7F).

During the experiment period, peroxidase (POD) activity progressively increased in upper leaves of 'PLRF' and 'Xingyu -2 ', and that of 'Xingyu-2' exhibited a more dramatic increase than in 'PLRF' (Figs. 8A and 8C), while POD activity of 'Xingyu-1' increased first and then decreased, drought stress obviously lowered POD activity at 21 days (Fig. 8B). Different responses of POD activity were seen in the lower leaves of edible canna cultivars (Figs. 8D to 8F). At early stage of drought stress, there existed little difference between the control and drought treatment in three cultivars, but after 28 days of drought stress, the POD activity decreased dramatically in 'PLRF' (Fig. 8D), changed little in 'Xingyu-1' (Fig. 8E),

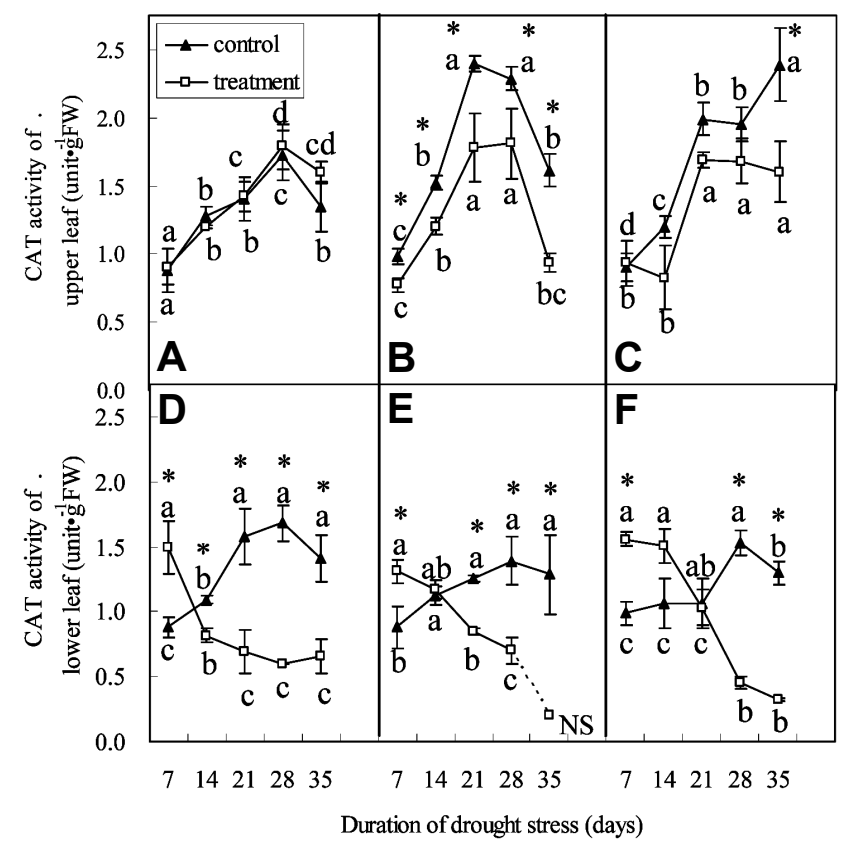

Fig. 7. Effects of drought stress on CAT activity in upper leaf $(A-C)$ and lower leaf (D-F) of edible canna 'PLRF' (AD), 'Xingyu-1' (BE), and 'Xingyu-2' (CF). but increased obviously in 'Xingyu-2' (Fig. 8F). When the treatment was up to 35 days, drought stress significantly decreased the POD activities in 'PLRF' and 'Xingyu-2' (Figs. $8 \mathrm{D}$ and $8 \mathrm{~F})$.

\section{The Correlation between Parameters}

The correlation analysis showed that the RWC showed significant positive correlation with Chl.a $(\mathrm{r}=0.811, p<$ $0.01)$, Chl.b $(\mathrm{r}=0.720, p<0.01)$, and CAT $(\mathrm{r}=0.572$, $p<0.01$ ), and significant negative correlation with MDA $(\mathrm{r}=-0.917, p<0.01)$ (Table 2). The MDA showed significant negative correlation with Chl.a $(\mathrm{r}=-720, p<0.01)$, Chl.b $(\mathrm{r}=-616, p<0.01)$, and CAT $(\mathrm{r}=-0.408, p<0.05)$. The Chl.a was positively correlated with Chl.b and CAT, the correlation coefficient was $0.806(p<0.01)$ and $0.531(p$ $<0.01$ ), respectively. There were obvious positive correlation between Chl.b and CAT ( $\mathrm{r}=0.697, p<0.01)$, between SOD and CAT $(r=0.399, p<0.05)$, and between SOD and POD

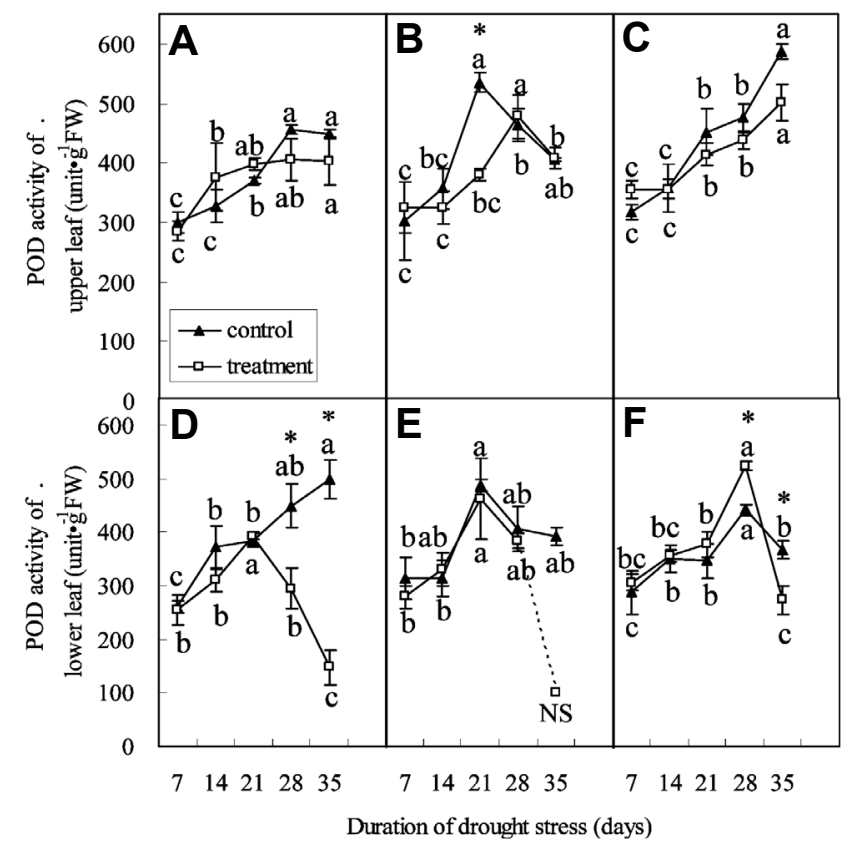

Fig. 8. Effects of drought stress on POD activity in upper leaf (A-C) and lower leaf (D-F) of edible canna 'PLRF' (AD), 'Xingyu-1' (BE), and 'Xingyu-2' (CF).

Table 2. Correlations of physiological index in leaves of edible canna 'PLRF', 'Xingyu-1', and 'Xingyu-2' under drought stress.

\begin{tabular}{|c|c|c|c|c|c|c|}
\hline Index & RWC & MDA & Chl.a & Chl.b & CAT & POD \\
\hline MDA & $-0.917^{* *}$ & & & & & \\
\hline Chl.a & $0.811^{* *}$ & $-0.720^{* *}$ & & & & \\
\hline Chl.b & $0.720^{* *}$ & $-0.616^{* *}$ & $0.806^{* *}$ & & & \\
\hline CAT & $0.572^{* *}$ & $-0.408^{*}$ & $0.531^{* *}$ & $0.697^{* *}$ & & \\
\hline POD & 0.242 & -0.114 & 0.120 & 0.164 & 0.292 & \\
\hline SOD & 0.055 & 0.102 & 0.039 & -0.001 & $0.399^{\star}$ & $0.693^{*}$ \\
\hline
\end{tabular}

${ }_{*, * \star}^{*}$ Significant correlations at $5 \%$ and at $1 \%$ level $(n=29)$, respectively, using Pearson correlation coefficients. 
$(\mathrm{r}=0.693, p<0.01)$. These results indicated that drought stress directly led to leaf water loss, and then increased the MDA content and decreased Chl.a and Chl.b content, whereas CAT, SOD and POD presumably played major roles in response to drought stress.

\section{Discussion}

Drought, as an abiotic stress, is one of the major factors to limit the plant growth and ecosystem production around the world (Xiao et al., 2009). In our study, the growth of the edible canna was inhibited, and senescence was accelerated by drought stress, especially in the lower leaves (Fig. 1). Previous studies also documented that visible syndromes of plant exposed to drought were leaf wilting, decrease of plant height, number and area of leaves (Talebi, 2009). It is known that plants evolve to avoid the stress with the maintenance of high tissue water potential either by reducing water loss or by improving water uptake or both (Farooq et al., 2009). This may be a strategy for plant to avoid stress by supplying water first to the upper functional leaves to lessen the consequence of drought on the function leaves. In our study, it was indicated that water deficit showed up in lower leaves with sustaining of drought stress (Fig. 2) and the photosynthesis of upper leaves obviously declined due to water loss under 35 days of drought stress (Table 1). Reduction of photosynthesis is first triggered by stomatal closure, resulting in limitation of ambient $\mathrm{CO}_{2}$ diffusion to the mesophyll and reduction of Ti (Ahmed et al., 2009; Grassi and Magnani, 2005). The WUE changed differently in edible canna when compared with the control, the WUE of 'Xingyu-2' showed a significant increase, 'PLRF' showed few change, while 'Xingyu-1' decreased obviously (Table 1). Drought-induced reductions of the photosynthetic pigment contents have been reported frequently (Elsheery and Cao, 2008; Loggini et al., 1999). In our study, in agreement with the plant morphological symptoms, photosynthetic pigment (Chl.a and Chl.b) contents decreased significantly under drought treatments in lower leaves of three cultivars (Figs. 3 and 4).

Drought stress is also known to enhance the production of reactive oxygen species (ROS), which could lead to the peroxidation of fatty acids in cell membranes (Foyer et al., 1994; Parkin et al., 1989). The Malondialdehyde (MDA) is the product of lipid peroxidation and usually used as a criterion to evaluate the stress-induced oxidative damage to membrane (Zhao and Tan, 2005). Low concentration of MDA has been associated with drought stress tolerance in some species, such as pea plants (Moran et al., 1994) and wheat (Sairam et al., 2000). In this study, the MDA contents increased progressively during our experiment period. Higher MDA contents were observed in 'Xingyu-1' and in lower leaves (Figs. 5A to $5 \mathrm{~F}$ ) indicated 'Xingyu-1' was relatively more sensitive to drought than 'PLRF' and 'Xingyu-2', and the oxidative stress was more severe in the lower leaves than in the upper leaves. This result was accordance with the results of Zhang et al. (2013). The increase of MDA contents (Figs. 5A to 5F) and the rapid degradation of chlorophyll (Figs. 3 and 4) was observed in edible canna plants exposed to drought stress. There were significant negative correlation between chl. content and MDA content, significant positive correlation between Chl.a, Chl.b and RWC. These results suggested drought stress directly induced oxidative damage and led to the destruction of pigments synthesis, and finally decreased the photosynthesis efficiency (Tables 1 and 2).

Fortunately, plants have the capacity to eliminate ROS with an efficient ROS-scavenging system. Antioxidant enzymes, such as SOD, CAT, and POD, constitute the major part of the plant antioxidant defense system. Under drought stress, antioxidant enzymes are directly involved in scavenging ROS and protecting plant against ROS damage (Xue and Liu, 2008), they are crucial for determining the steady-state level of ROS (Habibi, 2012; Mittler, 2002; van Rensburg and Kruger, 1994; Yang et al., 2008). In this study, drought stress changed the activities of SOD, CAT, and POD in edible canna. In upper leaves, the SOD activity under drought stress was still as high as or even higher than the control in upper leaves of three cultivars (Figs. 6A to 6C), the CAT activity showed little difference between two treatments of 'PLRF' and 'Xingyu-2' from 7 days to 35 days (Figs. 7A to 7C), except 'Xingyu-2' at 35 days, but that of 'Xingyu-1' significantly decreased due to drought stress (Fig. 7B), and the POD activity of 'Xingyu-2' progressively increased and has no difference between two treatments (Fig. 8C), that of 'PLRF' also increased, but the increase rate was lower than 'Xingyu-2' (Fig. 8A), the POD activity of 'Xingyu- 1 ' increased first and then decreased sharply in both treatments, and was obviously decreased by 21 days of drought stress (Fig. 8B). In lower leaves, the effect of water stress was more pronounced (Figs. 6 to 8). There were obvious linear trends between RWC, MDA, and CAT. So we could conclude that SOD, CAT and POD appeared to play an important role in resisting oxidative stress induced by water deficit, and associated with the better protection against oxidative damage in 'Xingyu-2' than in 'PLRF' and 'Xingyu-1'. These results indicated that 'Xingyu-2' was more drought tolerant than the 'PLRF' and 'Xingyu-1' by maintaining higher antioxidant enzymes. The similar results were observed in marigold (Tian et al., 2012), rice (Guo et al., 2006), cabbage (Singh et al., 2010), wheat (Simova-Stoilova et al., 2009) and white clover (Wang et al., 2008). The balance between SOD and CAT or POD 
activities in cells is importance for ROS scavenging (Mathur et al., 2009). In our study, there were significant positive correlations between SOD, POD, and CAT. It was noted that the synergistic reactions of SOD-CAT-POD system effectively scavenged oxidative damage in edible canna under drought stress. This result was accordance with the results of Tian et al. (2012) and Han et al. (2010).

In conclusion, our results showed that 'Xingyu-2' appeared to be more resistant to drought stress because 'Xingyu-2' expressed lower wilting index at the same stress time and could tolerate more time under drought stress than 'PLRF' and 'Xingyu-1'. In agreed with the wilting index and morphological symptoms, 'Xingyu-2' retained higher RWC and lower MDA content due to high antioxidant enzyme activities and higher WUE, especially in the lower leaves during early drought stress stage. These results could help us understand the response mechanism of edible canna genotypes to drought stress. It may contribute to select drought-resistant cultivars or germplasm based on these criteria, which will benefit cultivation and production of edible canna.

\section{Literature Cited}

Adhanom, G.T., R.J. Strirzaker, S.A. Lorentz, J.G. Annandale, and J.M. Steyn. 2012. Comparison of methods for determining unsaturated hydraulic conductivity in the wet range to evaluate the sensitivity of wetting front detectors. Water SA 38:67-75.

Ahmed, C.B., B.B. Rouina, S. Sensoy, M. Boukhris, and F.B. Abdallah. 2009. Changes in gas exchange, proline accumulation and antioxidative enzyme activities in three olive cultivars under contrasting water availability regimes. Environ. Exp. Bot. 67:345-352.

Basu, S., A. Roychoudhury, P.P. Saha, and D.N. Sengupta. 2010. Differential antioxidative responses of indica rice cultivars to drought stress. Plant Growth Regul. 60:51-59.

Boyer, J.S. 1982. Plant productivity and environment. Science 218:443-448.

Chance, B. and A.C. Maehly. 1955. Assay of catalase and perxoidase, p. 764-775. In: S.P. Colowick and N.O. Kaplan (eds.). Methods in enzymology. Academic Press, New York.

Chaves, M.M. 1991. Effects of water deficits on carbon assimilation. J. Exp. Bot. 42:1-16.

Chaves, M.M. and M.M. Oliveira. 2004. Mechanisms underlying plant resilience to water deficits: Prospects for water-saving agriculture. J. Exp. Bot. 55:2365-2384.

Cornic, G. 1994. Drought stress and high light effects on leaf photosynthesis, p. 297-313. In: N.R. Baker and J.R. Bowyer (eds.). Photoinhibition of photosynthesis from molecular mechanisms to the field. BIOS Scientific Publishers, Oxford.

Elsheery, N.I. and K.F. Cao. 2008. Gas exchange, chlorophyll fluorescence, and osmotic adjustment in two mango cultivars under drought stress. Acta Physiol. Plant. 30:769-777.

Ennahli, S. and H.J. Earl. 2005. Physiological limitations to photosynthetic carbon assimilation in cotton under water stress.
Crop Sci. 45:2374-2382.

Farooq, M., A. Wahid, N. Kobayashi, D. Fujita, and S.M.A. Basra. 2009. Plant drought stress: Effects, mechanisms and management. Agron. Sustain Dev. 29:185-212.

Foyer, C.H., M. Lelandais, and K.J. Kunert. 1994. Photooxidative stress in plants. Plant Physiol. 92:696-717.

Giannopolities, C.H. and S.K. Ries. 1977. Superoxide dismutases: I. Occurrence in higher plants. Plant Physiol. 59:309-314.

Gobin, A. 2012. Impact of heat and drought stress on arable crop production in Belgium. Nat. Hazards Earth Syst. Sci. 12:1911-1922.

Grassi, G. and F. Magnani. 2005. Stomatal, mesophyll conductance and biochemical limitations to photosynthesis as affected by drought and leaf ontogeny in ash and oak trees. Plant Cell Environ. 28:834-849.

Guo, Z., W. Ou, S. Lu, and Q. Zhong. 2006. Differential responses of antioxidative system to chilling and drought in four rice cultivars differing in sensitivity. Plant Physiol. Biochem. 44: 828-836.

Habibi, G. 2012. Exogenous salicylic acid alleviates oxidative damage of barley plants under drought stress. Acta Biologica Szegediensis 56:57-63.

Han, G., Q. Dang, and Z. Zhao. 2010. Responses of antioxidation protective system of Caragana korshinskii Kom. to drought stress. Acta Agrestia Sinica 18:528-532.

Heath, R.L. and L. Packer. 1968. Photoperoxidation in isolated chloroplast I. Kinetics and stoichiometry of fatty acid peroxidation. Arch Biochem. Biophys. 25:189-198.

Jones, H.G. and J.E. Corlett. 1992. Current topics in drought physiology. J. Agric. Sci. 119:291-296.

Kato, M. and K. Imai. 1996. Studies on matter production of edible canna (Canna edulis Ker.). IV. Leaf unrolling and changes in leaf photosynthetic rates with growth under field conditions. Japan. J. Crop Sci. 65:253-259.

Lares, M. and E. Pérez. 2006. Determination of the mineral fraction and rheological properties of microwave modified starch from Canna Edulis. Plant Food Hum. Nutr. 61:109-113.

Lawlor, D.W. 1995. The effects of water deficit on photosynthesis, p. 129-160. In: N. Smirnoff (ed.). Environment and plant metabolism. Flexibility and acclimation. BIOS Scientific Publishers, Oxford.

Li, F., X.Y. Qin, Y.H. Xie, X.S. Chen, J.Y. Hu, Y.Y. Liu, and Z.Y. Hou. 2013. Physiological mechanisms for plant distribution pattern: Responses to flooding and drought in three wetland plants from Dongting Lake, China. Limnology 14:71-76.

Li, L., N.H. Li, S.M. Jiang, J.Y. Leng, and X.J. Wang. 2009. Plant physiology module test guidance. Science Press, Beijing.

Lichtenthaler, H.K. and A.R. Wellburn. 1983. Determination of total carotenoids and chlorophyll $\mathrm{a}$ and $\mathrm{b}$ of leaf extracts in different solvents. Biochem. Soc. Trans. 603:591-592.

Lii, C.Y. and Y.H. Chang. 1991. Study of starch in Taiwan. Food Rev. Inter. 7:185-203.

Loggini, B.A., Scartazza, E. Brugnoli, and F. Navazari-Izzo. 1999. Antioxidative defense system, pigment composition, and photosynthetic efficiency in two wheat cultivars subjected to drought. Plant Physiol. 119:1091-1099.

Mafakheri, A., A. Siosemardeh, B. Bahramnejad, P.C. Struik, and E. Sohrabi. 2010. Effect of drought stress on yield, proline 
and chlorophyll contents in three chickpea cultivars. Austr. J. Crop Sci. 4:580-585.

Mathur, P.B., M.J. Devi, V. Vadez, and K.K. Sharma. 2009. Differential antioxidative responses in transgenic peanut bear no relationship to their superior transpiration efficiency under drought stress. J. Plant Physiol. 166:1207-1217.

Mittler, R. 2002. Oxidative stress, antioxidants and stress tolerance. Trends Plant Sci. 7:405-410.

Moorthy, S.N., B. Vimala, and A. Mukherjee. 2002. Physicochemical and functional properties of Canna edulis starch. Trop. Sci. 42:75-77.

Moran, J.F., M. Becana, I. Iturbe-Ormaetxe, S. Frechilla, R.V. Klucas, and P. Aparicio-Tejo. 1994. Drought induces oxidative stress in pea plants. Planta 194:346-352.

Ozkur, O., F. Ozdemir, M. Bor, and I. Turkan. 2009. Physiochemical and antioxidant responses of the perennial xerophyte Capparis ovata Desf. to drought. Environ. Exp. Bot. 66:487-492.

Pan, X.J., D.Y. Li, and W.E. Zhang. 2011. Photosynthesis characteristics of wild Vitis species native in Guizhou Province. J. Plant Genet. Resources 12:145-149.

Parkin, K.L., A. Marangoni, R. Jackman, R. Yada, and D. Stanley. 1989. Chilling injury. A review of possible mechanisms. J. Food Biochem. 13:127-153.

Pérez, E. and M. Lares. 2005. Chemical composition, mineral profile, and functional properties of canna (Canna edulis) and arrowroot (Maranta spp.) starches. Plant Foods Hum. Nutr. 60:113-116.

Pérez, E., M. Lares, and Z. González. 1997. Some characteristics of Sagu (Canna edulis Ker.) and Zulu (Maranta sp.) rhizomes. J. Agric. Food Chem. 45:2546-2549.

Pérez, E., W.M. Breene, and Y.A. Bahnassey. 1998. Gelatinization profiles of Peruvian carrot, cocoyam and potato starches as measured with the Brabender viscoamylograph, rapid viscoanalyzer, and differential scanning calorimeter. Starch 50:14-16.

Sairam, R.K., G.C. Srivastava, and D.C. Saxena. 2000. Increased antioxidant activity under elevated temperatures: A mechanism of heat stress tolerance in wheat genotypes. Biol. Plant. 43: 245-251.

Sanchez-Rodriguez, E., M. Rubio-Wilhelmi, M. Cervilla, B. Blasco, J.J. Rios, M.A. Rosales, L. Romero, and J.M. Ruiz. 2010. Genotypic differences in some physiological parameters symptomatic for oxidative stress under moderate drought in tomato plants. Plant Sci. 178:30-40.

Simova-Stoilova, L., K. Demirevska, T. Petrova, N. Tsenov, and U. Feller. 2009. Antioxidative protection and proteolytic activity in tolerant and sensitive wheat (Triticuma estivum L.) varieties subjected to long-term field drought. Plant Growth Regul. 58:107-117.

Singh, B.K., S.R. Sharma, and B. Singh. 2010. Antioxidant enzymes in cabbage: Variability and inheritance of superoxide dismutase, peroxidase and catalase. Sci. Hort. 124:9-13.

Sriroth, K., K. Piyachomkwan, S. Jin, and C.G. Oates. 2001. Canna starch: Properties, processing and utilization. In 52th Starch Convention. Association of Cereal Research. April 25-27, 2001, Detmold, Germany.

Talebi, R. 2009. Effective selection criteria for assessing drought stress tolerance in durum wheat (Triticum durum Desf.). Gen. Appl. Plant Physiol. 35:64-74.
Thitipraphunkul, K., D. Uttapap, K. Piyachomkwan, and Y. Takeda. 2003a. A comparative study of edible canna (Canna edulis) starch from different cultivars. Part I. Chemical composition and physicochemical properties. Carbohydr. Polym. 53:317-324.

Thitipraphunkul, K., D. Uttapap, K. Piyachomkwan, and Y. Takeda. 2003b. A comparative study of edible canna (Canna edulis) starch from different cultivars. Part II. Molecular structure of amylase and amylopectin. Carbohydr. Polym. 54:489-498.

Tian Z.G., F. Wang, W.E. Zhang, C.M. Liu, and X.M. Zhao. 2012. Antioxidant mechanism and lipid peroxidation in leaves and petals of Marigold in response to drought stress. Hort. Environ. Biotechnol. 53:183-192.

Türkan, I., M. Bor, F. Ozdemir, and H. Koca. 2005. Differential responses of lipid peroxidation and antioxidants in the leaves of drought tolerant $P$. acutifolius Gray and drought sensitive $P$. vulgaris $\mathrm{L}$. subjected to PEG mediated water stress. Plant Sci. 168:223-231.

van Rensburg, L. and G.H.J. Kruger. 1994. Evaluation of components of oxidative stress metabolism for use in selection of drought tolerant cultivars of Nicotiana tabacum L. J. Plant Physiol. 143:730-737.

Wang, C.Q. and R.C. Li. 2008. Enhancement of superoxide dismutase activity in the leaves of white clover (Trifolium repens L.) in response to polyethylene glycol-induced water stress. Acta Physiol. Plant. 30:841-847.

Weatherley, P.E. 1950. Studies in the water relations of the cotton plants. I. The field measurements of water deficits in leaves. New Phytol. 49: 81-87.

Wootton, M. and M. Bamunuarachchi. 1978. Water binding capacity of commercial produced native and modified starches. Starch 30:306-309.

Wu, T.X., K.C. Zhang, and C.Z. Liu. 2005. Study on the property of Canna edulis Ker. its application in ethanol fermentation. Liquor-making Sci. Tech. 136:40-42.

Xiao, X.W., F. Yang, S. Zhang, H. Korpelainen, and C.Y. Li. 2009. Physiological and proteomic responses of two contrasting Populus cathayana populations to drought stress. Physiol. Plant. 136:150-168.

Xue, Y.F. and Z.P. Liu. 2008. Antioxidant enzymes and physiological characteristics in two Jerusalem artichoke cultivars under salt stress. Russ. J. Plant Physiol. 55:776-781.

Yang, Y., C. Han, Q. Liu, B. Lin, and J. Wang. 2008. Effect of drought and low light on growth and enzymatic antioxidant system of Picea asperata seedlings. Acta Physiol. Plant. 30: 433-440.

Yun, Y.S., M. Satake, S. Katsuki, and A. Kunugi. 2004. Phenylpropanoid derivatives from edible canna, Canna edulis. Phytochemistry 65:2167-2171

Zhang, M.D., Q. Chen, and S.H. Shen. 2011. Physiological responses of two Jerusalem artichoke cultivars to drought stress induced by polyethylene glycol. Acta Physiol. Plant. 33:313-318.

Zhang, W.E., Z.G. Tian, X,J. Pan, X.M. Zhao, and F. Wang. 2013. Oxidative stress and non-enzymatic antioxidants in leaves of three edible canna cultivars under drought stress. Hort. Environ. Biotechnol. 54:1-8.

Zhao, H.J. and J.F. Tan. 2005. Role of calcium ion in protection against heat and high irradiance stress-induced oxidative damage to photosynthesis of wheat leaves. Photosynthetica 43:473-476. 